\title{
Causes and Explanations: A Structural-Model Approach. Part II: Explanations
}

\author{
Joseph Y. Halpern* \\ Cornell University \\ Dept. of Computer Science \\ Ithaca, NY 14853 \\ halpern@cs.cornell.edu \\ http://www.cs.cornell.edu/home/halpern \\ Judea Pearl ${ }^{\dagger}$ \\ Dept. of Computer Science \\ University of California, Los Angeles \\ Los Angeles, CA 90095 \\ judea@cs.ucla.edu \\ http://www.cs.ucla.edu/ judea
}

February 1, 2008

\begin{abstract}
We propose new definitions of (causal) explanation, using structural equations to model counterfactuals. The definition is based on the notion of actual cause, as defined and motivated in a companion paper. Essentially, an explanation is a fact that is not known for certain but, if found to be true, would constitute an actual cause of the fact to be explained, regardless of the agent's initial uncertainty. We show that the definition handles well a number of problematic examples from the literature.
\end{abstract}

\footnotetext{
*Supported in part by NSF under grants IRI-96-25901 and 0090145.

†Supported in part by grants from NSF, ONR, AFOSR, and MICRO.
} 


\section{Introduction}

The automatic generation of adequate explanations is a task essential in planning, diagnosis and natural language processing. A system doing inference must be able to explain its findings and recommendations to evoke a user's confidence. However, getting a good definition of explanation is a notoriously difficult problem, which has been studied for years. (See [Chajewska and Halpern 1997; Gärdenfors 1988; Hempel 1965; Pearl 1988; Salmon 1989] and the references therein for an introduction to and discussion of the issues.)

In Part I of this paper [Halpern and Pearl 2004] we give a definition of actual causality using structural equations. Here we show how the ideas behind that definition can be used to give an elegant definition of (causal) explanation that deals well with many of the problematic examples discussed in the literature. The basic idea is that an explanation is a fact that is not known for certain but, if found to be true, would constitute an actual cause of the explanandum (the fact to be explained), regardless of the agent's initial uncertainty.

Note that our definition involves causality and knowledge. Following Gärdenfors [1988], we take the notion of explanation to be relative to an agent's epistemic state. What counts as an explanation for one agent may not count as an explanation for another agent. We also follow Gärdenfors in allowing explanations to include (fragments of) a causal model. To borrow an example from Gärdenfors, an agent seeking an explanation of why Mr. Johansson has been taken ill with lung cancer will not consider the fact that he worked for years in asbestos manufacturing a part of an explanation if he already knew this fact. For such an agent, an explanation of Mr. Johansson's illness may include a causal model describing the connection between asbestos fibers and lung cancer. On the other hand, for someone who already knows the causal model but does not know that Mr. Johansson worked in asbestos manufacturing, the explanation would involve Mr. Johansson's employment but would not mention the causal model.

Where our definition differs from that of Gärdenfors (and all others in the literature) is in the way it is formulated in terms of the underlying notions of knowledge, causal models, actual causation, and counterfactuals. The definition is not based on probabilistic dependence, "statistical relevance", or logical implication, and thus is able to deal with the directionality inherent in common explanations. While it seems reasonable to say "the height of the flag pole explains the length of the shadow", it would sound awkward if one were to explain the former with the latter. Our definition is able to capture this distinction easily.

The best judge of the adequacy of an approach are the intuitive appeal of the definitions and how well it deals with examples; we believe that this paper shows that our approach fares well on both counts.

The remainder of the paper is organized as follows. In Section 2, we review the basic definitions of causal models based on structural equations, which are the basis for 
our definitions of causality and explanation, and then review the definition of causality from the companion paper. We have tried to include enough detail here to make the paper self-contained, but we encourage the reader to consult the companion paper for more motivation and discussion. In Section 3 we give the basic definition of explanation, under the assumption that the causal model is known. In Section 4, probability is added to the picture, to give notions of partial explanation and explanatory power. The general definition, which dispenses with the assumption that the causal model is known, is discussed in Section 5. We conclude in Section 6 with some discussion.

\section{Causal Models and the Definition of Actual Causal- ity: A Review}

To make this paper self-contained, this section repeats material from the companion paper; we review the basic definitions of causal models, as defined in terms of structural equations, the syntax and semantics of a language for reasoning about causality and explanations, and the definition of actual cause.

\subsection{Causal models}

The use of structural equations as a model for causal relationships is standard in the social sciences, and seems to go back to the work of Sewall Wright in the 1920s (see [Goldberger 1972] for a discussion); the particular framework that we use here is due to Pearl [1995], and is further developed in [Pearl 2000].

The basic picture is that the world is described by random variables, some of which may have a causal influence on others. This influence is modeled by a set of structural equations. Each equation represents a distinct mechanism (or law) in the world, which may be modified (by external actions) without altering the others. In practice, it seems useful to split the random variables into two sets, the exogenous variables, whose values are determined by factors outside the model, and the endogenous variables, whose values are ultimately determined by the exogenous variables. It is these endogenous variables whose values are described by the structural equations.

Formally, a signature $\mathcal{S}$ is a tuple $(\mathcal{U}, \mathcal{V}, \mathcal{R})$, where $\mathcal{U}$ is a set of exogenous variables, $\mathcal{V}$ is a finite set of endogenous variables, and $\mathcal{R}$ associates with every variable $Y \in \mathcal{U} \cup \mathcal{V}$ a nonempty set $\mathcal{R}(Y)$ of possible values for $Y$ (that is, the set of values over which $Y$ ranges). A causal (or structural) model over signature $\mathcal{S}$ is a tuple $M=(\mathcal{S}, \mathcal{F})$, where $\mathcal{F}$ associates with each variable $X \in \mathcal{V}$ a function denoted $F_{X}$ such that $F_{X}$ : $\left(\times_{U \in \mathcal{U}} \mathcal{R}(U)\right) \times\left(\times_{Y \in \mathcal{V}-\{X\}} \mathcal{R}(Y)\right) \rightarrow \mathcal{R}(X) . \quad F_{X}$ determines the value of $X$ given the values of all the other variables in $\mathcal{U} \cup \mathcal{V}$. For example, if $F_{X}(Y, Z, U)=Y+U$ (which we usually write as $X=Y+U)$, then if $Y=3$ and $U=2$, then $X=5$, regardless of how $Z$ is set. 
These equations can be thought of as representing processes (or mechanisms) by which values are assigned to variables. Hence, like physical laws, they support a counterfactual interpretation. For example, the equation above claims that, in the context $U=u$, if $Y$ were 4 , then $X$ would be $u+4$ (which we write as $(M, u) \models[Y \leftarrow 4](X=u+4)$ ), regardless of what values $X, Y$, and $Z$ actually take in the real world.

The counterfactual interpretation and the causal asymmetry associated with the structural equations are best seen when we consider external interventions (or spontaneous changes), under which some equations in $F$ are modified. An equation such as $x=F_{X}(\vec{u}, y)$ should be thought of as saying that in a context where the exogenous variables have values $\vec{u}$, if $Y$ were set to $y$ by some means (not specified in the model), then $X$ would take on the value $x$, as dictated by $F_{X}$. The same does not hold when we intervene directly on $X$; such an intervention amounts to assigning a value to $X$ by external means, thus overruling the assignment specified by $F_{X}$.

For those more comfortable with thinking of counterfactuals in terms of possible worlds, this modification of equations may be given a simple "closest world" interpretation: the solution of the equations obtained replacing the equation for $Y$ with the equation $Y=y$, while leaving all other equations unaltered, gives the closest "world" to the actual world where $Y=y$.

We can describe (some salient features of) a causal model $M$ using a causal network. This is a graph with nodes corresponding to the random variables in $\mathcal{V}$ and an edge from a node labeled $X$ to one labeled $Y$ if $F_{Y}$ depends on the value of $X$. Intuitively, variables can have a causal effect only on their descendants in the causal network; if $Y$ is not a descendant of $X$, then a change in the value of $X$ has no effect on the value of $Y$. Causal networks are similar in spirit to Lewis's neuron diagrams [1973], but there are significant differences as well (see Part I for a discussion). In this paper, we restrict attention to what are called recursive (or acyclic) equations; these are ones that can be described with a causal network that is a directed acyclic graph (that is, a graph that has no cycle of edges). It should be clear that if $M$ is a recursive causal model, then there is always a unique solution to the equations in $M$, given a setting $\vec{u}$ for the variables in $\mathcal{U}{ }^{1}$ Such a setting is called a context. Contexts will play the role of possible worlds when we model uncertainty. For future reference, a pair $(M, \vec{u})$ consisting of a causal model and a context is called a situation.

Example 2.1: Suppose that two arsonists drop lit matches in different parts of a dry forest, and both cause trees to start burning. Consider two scenarios. In the first, called the disjunctive scenario, either match by itself suffices to burn down the whole forest. That is, even if only one match were lit, the forest would burn down. In the second scenario, called the conjunctive scenario, both matches are necessary to burn down the forest; if only one match were lit, the fire would die down before the forest was consumed.

\footnotetext{
${ }^{1}$ In the companion paper, there is some discussion on how to extend the definition of causality to nonrecursive models. It seems that these ideas should apply to our definition of explanation as well. However, we do not pursue this issue here.
} 
We can describe the essential structure of these two scenarios using a causal model with four variables:

- an exogenous variable $U$ that determines, among other things, the motivation and state of mind of the arsonists. For simplicity, assume that $\mathcal{R}(U)=\left\{u_{00}, u_{10}, u_{01}, u_{11}\right\}$; if $U=u_{i j}$, then the first arsonist intends to start a fire iff $i=1$ and the second arsonist intends to start a fire iff $j=1$. In both scenarios $U=u_{11}$.

- endogenous variables $M L_{1}$ and $M L_{2}$, each either 0 or 1 , where $M L_{i}=0$ if arsonist $i$ doesn't drop the match and $M L_{i}=1$ if he does, for $i=1,2$.

- an endogenous variable $F B$ for forest burns down, with values 0 (it doesn't) and 1 (it does).

Both scenarios have the same causal network (see Figure 1); they differ only in the equation for $F B$. For the disjunctive scenario we have $F_{F B}(u, 1,1)=F_{F B}(u, 0,1)=$ $F_{F B}(u, 1,0)=1$ and $F_{F B}(u, 0,0)=0$ (where $u \in \mathcal{R}(U)$ ); for the conjunctive scenario we have $F_{F B}(u, 1,1)=1$ and $F_{F B}(u, 0,0)=F_{F B}(u, 1,0)=F_{F B}(u, 0,1)=0$.

In general, we expect that the causal model for reasoning about forest fires would involve many other variables; in particular, variables for other potential causes of forest fires such as lightning and unattended campfires. Here we focus on that part of the causal model that involves forest fires started by arsonists. Since for causality we assume that all the relevant facts are given, we can assume here that it is known that there were no unattended campfires and there was no lightning, which makes it safe to ignore that portion of the causal model.

Denote by $M_{1}$ and $M_{2}$ the (portion of the) causal models associated with the disjunctive and conjunctive scenarios, respectively. The causal network for the relevant portion of $M_{1}$ and $M_{2}$ is described in Figure 1. The diagram emphasizes that the value of $F B$

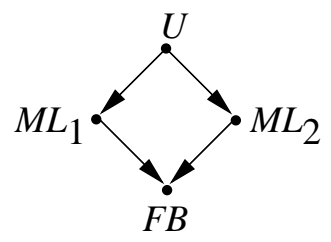

Figure 1: The causal network for $M_{1}$ and $M_{2}$.

is determined by the values of $M L_{1}$ and $M L_{2}$ (which in turn are determined by the value of the exogenous variable $U$.

As we said, a causal model has the resources to determine counterfactual effects. Given a causal model $M=(\mathcal{S}, \mathcal{F})$, a (possibly empty) vector $\vec{X}$ of variables in $\mathcal{V}$, and vectors $\vec{x}$ and $\vec{u}$ of values for the variables in $\vec{X}$ and $\mathcal{U}$, respectively, we can define a new causal model denoted $M_{\vec{X} \leftarrow \vec{x}}$ over the signature $\mathcal{S}_{\vec{X}}=\left(\mathcal{U}, \mathcal{V}-\vec{X},\left.\mathcal{R}\right|_{\mathcal{V}-\vec{X}}\right)$. Formally, 
$M_{\vec{X} \leftarrow \vec{x}}=\left(\mathcal{S}_{\vec{X}}, \mathcal{F}^{\vec{X} \leftarrow \vec{x}}\right)$, where $F_{Y}^{\vec{X} \leftarrow \vec{x}}$ is obtained from $F_{Y}$ by setting the values of the variables in $\vec{X}$ to $\vec{x}$. Intuitively, this is the causal model that results when the variables in $\vec{X}$ are set to $\vec{x}$ by some external action that affects only the variables in $\vec{X}$; we do not model the action or its causes explicitly. For example, if $M_{1}$ is the model for the disjunctive scenario in Example 2.1, then $\left(M_{1}\right)_{M L_{1} \leftarrow 0}$ is the model where $F B=M L_{2}$ : if the first match is not dropped, then there is a fire if and only if the second match is dropped. Similarly, $\left(M_{2}\right)_{M L_{2} \leftarrow 0}$ is the model where $F B=0$ : if the first match is not dropped, then there is no fire in the conjunctive scenario. Note that if $M$ is a recursive causal model, then there is always a unique solution to the equations in $M_{\vec{X} \leftarrow \vec{x}}$ for all $\vec{X}$ and $\vec{x}$.

\subsection{Syntax and Semantics:}

Given a signature $\mathcal{S}=(\mathcal{U}, \mathcal{V}, \mathcal{R})$, a formula of the form $X=x$, for $X \in V$ and $x \in \mathcal{R}(X)$, is called a primitive event. A basic causal formula is one of the form $\left[Y_{1} \leftarrow y_{1}, \ldots, Y_{k} \leftarrow\right.$ $\left.y_{k}\right] \varphi$, where

- $\varphi$ is a Boolean combination of primitive events;

- $Y_{1}, \ldots, Y_{k}$ are distinct variables in $\mathcal{V}$; and

- $y_{i} \in \mathcal{R}\left(Y_{i}\right)$.

Such a formula is abbreviated as $[\vec{Y} \leftarrow \vec{y}] \varphi$. The special case where $k=0$ is abbreviated as $\varphi$. Intuitively, $\left[Y_{1} \leftarrow y_{1}, \ldots, Y_{k} \leftarrow y_{k}\right] \varphi$ says that $\varphi$ holds in the counterfactual world that would arise if $Y_{i}$ were set to $y_{i}, i=1, \ldots, k$. A causal formula is a Boolean combination of basic causal formulas. ${ }^{2}$

A causal formula $\varphi$ is true or false in a causal model, given a context. We write $(M, \vec{u}) \models \varphi$ if $\varphi$ is true in causal model $M$ given context $\vec{u} . \quad(M, \vec{u}) \models[\vec{Y} \leftarrow \vec{y}](X=x)$ if the variable $X$ has value $x$ in the unique (since we are dealing with recursive models) solution to the equations in $M_{\vec{Y} \leftarrow \vec{y}}$ in context $\vec{u}$ (that is, the unique vector of values for the exogenous variables that simultaneously satisfies all equations $F_{Z}^{\vec{Y} \leftarrow \vec{y}}, Z \in \mathcal{V}-\vec{Y}$, with the variables in $\mathcal{U}$ set to $\vec{u}$ ). We extend the definition to arbitrary causal formulas in the obvious way.

Thus, in Example 2.1, we have $\left(M_{1}, u_{11}\right) \models\left[M L_{1}=0\right](F B=1)$ and $\left(\mathcal{M}_{2}, u_{11}\right) \models$ $\left[M L_{1}=0\right](F B=0)$. In the disjunctive model, in the context where both arsonists drop a match, if the first arsonist does not drop a match, the forest still burns down. On the

\footnotetext{
${ }^{2}$ If we write $\rightarrow$ for conditional implication, then a formula such as $[Y \leftarrow y] \varphi$ can be written as $Y=y \rightarrow \varphi$ : if $Y$ were $y$, then $\varphi$ would hold. We use the present notation to emphasize the fact that, although we are viewing $Y \leftarrow y$ as a modal operator, we are not giving semantics using the standard possible-worlds approach.
} 
other hand, in the conjunctive model, if the first arsonist does not drop a match (in the same context), the forest does not burn down.

Note that the structural equations are deterministic. We later add probability to the picture by putting a probability on the set of contexts (i.e., on the possible worlds). This probability is not needed in the definition of causality, but will be useful in the discussion of explanation.

\subsection{The Definition of Cause}

With all this notation in hand, we can now give a definition of actual cause ("cause" for short). We want to make sense out of statements of the form "event $A$ is an actual cause of event $\varphi$ (in context $\vec{u}$ )" ${ }^{3}$ The picture here is that the context (and the structural equations) are given. Intuitively, they encode the background knowledge. All the relevant events are known. The only question is picking out which of them are the causes of $\varphi$ or, alternatively, testing whether a given set of events can be considered the cause of $\varphi$.

The types of events that we allow as actual causes are ones of the form $X_{1}=x_{1} \wedge$ $\ldots \wedge X_{k}=x_{k}$ - that is, conjunctions of primitive events; we typically abbreviate this as $\vec{X}=\vec{x}$. The events that can be caused are arbitrary Boolean combinations of primitive events. We do not believe that we lose much by disallowing disjunctive causes here. Disjunctive explanations, however, are certainly of interest.

Roughly speaking, $\vec{X}=\vec{x}$ is a sufficient cause of $\varphi$ in situation $(M, \vec{u})$ if (a) $\vec{X}=\vec{x} \wedge \varphi$ is true in $(M, \vec{u})$ and (b) there is some set $\vec{x}^{\prime}$ of values of $\vec{X}$ and some other variables $\vec{W}$ (satisfying some constraints) such that setting $\vec{W}$ to $\vec{w}^{\prime}$ and changing $\vec{X}$ to $\vec{x}^{\prime}$ results in $\neg \varphi$ being true (that is, $\left.(M, \vec{u}) \models\left[\vec{X} \leftarrow \vec{x}^{\prime}, \vec{W} \leftarrow \vec{w}^{\prime}\right] \neg \varphi\right)$. Part (b) is very close to the standard counterfactual definition of causality, advocated by Lewis [1973] and others: $\vec{X}=\vec{x}$ is the cause of $\varphi$ if, had $\vec{X}$ not been equal to $\vec{x}, \varphi$ would not have been the case. The difference is that we allow setting some other variables $\vec{W}$ to $\vec{w}$. As we said, the formal definition puts some constraints on $\vec{W}$. Among other things, it must be the case that setting $\vec{X}$ to $\vec{x}$ is enough to force $\varphi$ to be true when $\vec{W}$ is set to $\vec{w}^{\prime}$. That is, it must be the case that $(M, \vec{u}) \models\left[\vec{X} \leftarrow \vec{x}, \vec{W} \leftarrow \vec{w}^{\prime}\right] \varphi$. (See the appendix for the formal definition.)

$\vec{X}=\vec{x}$ is an actual cause of $\varphi$ in $(M, \vec{u})$ if it is a sufficient clause with no irrelevant conjuncts. That is, $\vec{X} \leftarrow \vec{x}$ is an actual cause of $\varphi$ in $(M, \vec{u})$ if it is a sufficient cause and no subset of $\vec{X}$ is also a sufficient cause. Eiter and Lukasiewicz [2002] and, independently, Hopkins [2001] have shown that actual causes are always single conjuncts. As we shall see, this is not the case for explanations.

Returning to Example 2.1, note that $M L_{1}=1$ is an actual cause of $F B=1$ in both the conjunctive and the disjunctive scenarios. This should be clear in the conjunctive

\footnotetext{
${ }^{3}$ Note that we are using the word "event" here in the standard sense of "set of possible worlds" (as opposed to "transition between states of affairs"); essentially we are identifying events with propositions.
} 
scenario. Setting $M L_{1}$ to 0 results in the forest not burning down. To see that $M L_{1}=1$ is also a cause in the disjunctive scenario, let $W$ be $M L_{2}$. Note that $\left(M_{1}, u_{00}\right) \models\left[M L_{1} \leftarrow\right.$ $\left.0, M L_{2} \leftarrow 0\right] F B=0$, so the counterfactual condition is satisfied. Moreover, $\left(M_{1}, u_{00}\right) \models$ $\left[M L_{1} \leftarrow 1, M L_{2} \leftarrow 0\right] F B=1$; that is, in the disjunctive scenario, if the first arsonist drops the match, that is enough to burn down the forest, no matter what the second arsonist does. In either scenario, both arsonists dropping a lit match constitutes a sufficient cause for the forest fire, as does the first arsonist dropping a lit match and sneezing. Given an actual cause, a sufficient cause can be obtained by adding arbitrary conjuncts to it.

Although each arsonist is a cause of the forest burning down in the conjunctive scenario, under reasonable assumptions about the knowledge of the agent wanting an explanation, each arsonist alone is not an explanation of the forest burning down. Both arsonists together provide the explanation in that case; identifying arsonist 1 would only trigger a further quest for the identity of her accomplice. In the disjunctive scenario, each arsonist alone is an explanation of the forest burning down.

We hope that this informal discussion of the definition of causality will suffice for readers who want to focus mainly on explanation. For completeness, we give the formal definitions of sufficient and actual cause in the appendix. The definition is motivated, discussed, and defended in much more detail in Part I, where it is also compared with other definitions of causality. In particular, it is shown to avoid a number of problems that have been identified with Lewis's account (e.g., see [Pearl 2000, Chapter 10]), such as commitment to transitivity of causes. For the purposes of this paper, we ask that the reader accept our definition of causality. We note that, to some extent, our definition of explanation is modular in its use of causality, in that another definition of causality could be substituted for the one we use in the definition of explanation (provided it was given in the same framework).

\section{Explanation: The Basic Definition}

As we said in the introduction, many definitions of causal explanation have been given in the literature. The "classical" approaches in the philosophy literature, such as Hempel's [1965] deductive-nomological model and Salmon's [1989] statistical relevance model (as well as many other approaches), because they are based on logical implication and probabilistic dependence, respectively, fail to exhibit the directionality inherent in common explanations. Despite all the examples in the philosophy literature on the need for taking causality and counterfactuals into account, and the extensive work on causality defined in terms of counterfactuals in the philosophy literature, as Woodward [2001] observes, philosophers have been reluctant to build a theory of explanation on top of a theory of causality. The concern seems to be one of circularity. In this section, we give a definition of explanation based on the definition of causality discussed in Section 2.3. Circularity is avoided because the definition of explanation invokes strictly formal features of a causal model that do not in turn depend on the notion of explanation. 
Our definition of causality assumed that the causal model and all the relevant facts were given; the problem was to figure out which of the given facts were causes. In contrast, the role of explanation is to provide the information needed to establish causation. Roughly speaking, we view an explanation as a fact that is not known for certain but, if found to be true, would constitute a genuine cause of the explanandum (fact to be explained), regardless of the agent's initial uncertainty. Roughly speaking, the role of explanation is to provide the information needed to establish causation. Thus, as we said in the introduction, what counts as an explanation depends on what one already knows (or believes - we largely blur the distinction between knowledge and belief in this paper). As a consequence, the definition of an explanation should be relative to the agent's epistemic state (as in Gärdenfors [1988]). It is also natural, from this viewpoint, that an explanation will include fragments of the causal model $M$ or reference to the physical laws underlying the connection between the cause and the effect.

The definition of explanation is motivated by the following intuitions. An individual in a given epistemic state $K$ asks why $\varphi$ holds. What constitutes a good answer to his question? A good answer must (a) provide information that goes beyond $K$ and (b) be such that the individual can see that it would, if true, be (or be very likely to be) a cause of $\varphi$. We may also want to require that (c) $\varphi$ be true (or at least probable). Although our basic definition does not insist on (c), it is easy to add this requirement.

How do we capture an agent's epistemic state in our framework? For ease of exposition, we first consider the case where the causal model is known and only the context is uncertain. (The minor modifications required to deal with the general case are described in Section 5.) In that case, one way of describing an agent's epistemic state is by simply describing the set of contexts the agent considers possible.

Definition 3.1: (Explanation) Given a structural model $M, \vec{X}=\vec{x}$ is an explanation of $\varphi$ relative to a set $\mathcal{K}$ of contexts if the following conditions hold:

EX1. $(M, \vec{u}) \models \varphi$ for each context $\vec{u} \in \mathcal{K}$. (That is, $\varphi$ must hold in all contexts the agent considers possible - the agent considers what she is trying to explain as an established fact.)

EX2. $\vec{X}=\vec{x}$ is a sufficient cause of $\varphi$ in $(M, \vec{u})$ for each $\vec{u} \in \mathcal{K}$ such that $(M, \vec{u}) \models \vec{X}=\vec{x}$.

EX3. $\vec{X}$ is minimal; no subset of $\vec{X}$ satisfies EX2.

EX4. $(M, \vec{u}) \models \neg(\vec{X}=\vec{x})$ for some $\vec{u} \in \mathcal{K}$ and $\left(M, \vec{u}^{\prime}\right) \models \vec{X}=\vec{x}$ for some $\vec{u}^{\prime} \in \mathcal{K}$. (This just says that the agent considers a context possible where the explanation is false, so the explanation is not known to start with, and considers a context possible where the explanation is true, so that it is not vacuous.)

Our requirement EX4 that the explanation is not known may seem incompatible with linguistic usage. Someone discovers some fact $A$ and says "Aha! That explains 
why $B$ happened." Clearly, $A$ is not an explanation of why $B$ happened relative to the epistemic state after $A$ has been discovered, since at that point $A$ is known. However, $A$ can legitimately be considered an explanation of $B$ relative to the epistemic state before $A$ was discovered. Interestingly, as we shall see, although there is a cause for every event $\varphi$ (although sometimes it may be the trivial cause $\varphi$ ), one of the effects of the requirement EX4 is that some events may have no explanation. This seems to us quite consistent with standard usage of the notion of explanation. After all, we do speak of "inexplicable events".

What does the definition of explanation tell us for the arsonist example? What counts as a cause is, as expected, very much dependent on the causal model and the agent's epistemic state. If the causal model has only arsonists as the cause of the fire, there are two possible explanations in the disjunctive scenario: (a) arsonist 1 did it and (b) arsonist 2 did it (assuming $\mathcal{K}$ consists of three contexts, where either 1 , 2, or both set the fire). In the conjunctive scenario, no explanation is necessary, since the agent knows that both arsonists must have lit a match if arson is the only possible cause of the fire (assuming that the agent considers the two arsonists to be the only possible arsonists). That is, if the two arsonists are the only possible cause of the fire and the fire is observed, then $\mathcal{K}$ can consist of only one context, namely, the one where both arsonists started the fire. No explanation can satisfy EX4 in this case.

Perhaps more interesting is to consider a causal model with other possible causes, such as lightning and unattended campfires. Since the agent knows that there was a fire, in each of the contexts in $\mathcal{K}$, at least one of the potential causes must have actually occurred. If there is a context in $\mathcal{K}$ where only arsonist 1 dropped a lit match (and, say, there was lightning), another where only arsonist 2 dropped a lit match, and a third where both arsonists dropped matches, then, in the conjunctive scenario, $M L_{1}=1 \wedge M L_{2}=1$ is an explanation of $F B=1$, but neither $M L_{1}=1$ nor $M L_{2}=1$ by itself is an explanation (since neither by itself is a cause in all contexts in $\mathcal{K}$ that satisfy the formula). On the other hand, in the disjunctive scenario, both $M L_{1}=1$ and $M L_{2}=1$ are explanations.

Consider the following example, due to Bennett (see [Sosa and Tooley 1993, pp. 222223]), which is analyzed in Part I.

Example 3.2: Suppose that there was a heavy rain in April and electrical storms in the following two months; and in June the lightning took hold. If it hadn't been for the heavy rain in April, the forest would have caught fire in May. The first question is whether the April rains caused the forest fire. According to a naive counterfactual analysis, they do, since if it hadn't rained, there wouldn't have been a forest fire in June. In our framework, it is not the case that the April rains caused the fire, but they were a cause of there being a fire in June, as opposed to May. This seems to us intuitively right.

The situation can be captured using a model with three endogenous random variables:

- $A S$ for "April showers", with two values - 0 standing for did not rain heavily in April and 1 standing for rained heavily in April; 
- ES for "electric storms", with four possible values: $(0,0)$ (no electric storms in either May or June), (1,0) (electric storms in May but not June), (0,1) (storms in June but not May), and (1,1) (storms in both April and May);

- and $F$ for "fire", with three possible values: 0 (no fire at all), 1 (fire in May), or 2 (fire in June).

We do not describe the context explicitly. Assume its value $\vec{u}$ is such that it ensures that there is a shower in April, there are electric storms in both May and June, there is sufficient oxygen, there are no other potential causes of fire (like dropped matches), no other inhibitors of fire (alert campers setting up a bucket brigade), and so on. That is, we choose $\vec{u}$ so as to allow us to focus on the issue at hand and to ensure that the right things happened (there was both fire and rain). We will not bother writing out the details of the structural equations - they should be obvious, given the story (at least, for the context $\vec{u}$ ); this is also the case for all the other examples in this section. The causal network is simple: there are edges from $A S$ to $F$ and from $E S$ to $F$. As observed in Part I, each of the following holds.

- $A S=1$ is a cause of the June fire $(F=2)$.

- $A S=1$ is not a cause of the fire $(F=1 \vee F=2)$. If $E S$ is set $(0,1),(1,0)$, or $(1,1)$, then there will be a fire (in either May or June) whether $A S=0$ or $A S=1$. On the other hand, if $E S$ is set to $(0,0)$, then there is no fire, whether $A S=0$ or $A S=1$.

- $E S=(1,1)$ is a cause of both $F=2$ and $(F=1 \vee F=2)$. Having electric storms in both May and June caused there to be a fire.

- $A S=1 \wedge E S=(1,1)$ is a sufficient cause of $F=2$; each individual conjunct is an actual cause.

Now consider the problem of explanation. Suppose that the agent knows that there was an electric storm, but does not know when, and does not know whether there were April showers. Thus, $\mathcal{K}$ consists of six contexts, one corresponding to each of the values $(1,0),(0,1)$, and $(1,1)$ of $E S$ and the values 0 and 1 of $A S$. Then it is easy to see that $A S=1$ is not an explanation of fire $(F=1 \vee F=2)$, since it is not a cause of fire in any context in $\mathcal{K}$. Similarly, $A S=0$ is not an explanation of fire. On the other hand, each of $E S=(1,1), E S=(1,0)$, and $E S=(0,1)$ is an explanation of fire.

Now suppose that we are looking for an explanation of the June fire. Then the set $\mathcal{K}$ can consist only of contexts compatible with there being a fire in June. Suppose that $\mathcal{K}$ consists of three contexts, one where $A S=1$ and $E S=(0,1)$, one where $A S=1$ and $E S=(1,1)$, and one where $A S=0$ and $E S=(0,1)$. In this case, each of $A S=1$, $E S=(0,1)$, and $E S=(1,1)$ is an explanation of the June fire. (In the case of $A S=1$, we need to consider the setting where $E S=(1,1)$.) 
Finally, if the agent knows that there was an electric storm in May and June and heavy rain in April (so that $\mathcal{K}$ consists of only one context), then there is no explanation of either fire or the fire in June. Formally, this is because it is impossible to satisfy EX4. Informally, this is because the agent already knows why there was a fire in June.

Note that, as for causes, we have disallowed disjunctive explanations. Here the motivation is less clear cut. It does make perfect sense to say that the reason that $\varphi$ happened is either $A$ or $B$ (but I don't know which). There are some technical difficulties with disjunctive explanations, which suggest philosophical problems. For example, consider the conjunctive scenario of the arsonist example again. Suppose that the structural model is such that the only causes of fire are the arsonists, lightning, and unattended campfires and that $\mathcal{K}$ consists of contexts where each of these possibilities is the actual cause of the fire. Once we allow disjunctive explanations, what is the explanation of fire? One candidate is "either there were two arsonists or there was lightning or there was an unattended campfire (which got out of hand)". But this does not satisfy EX4, since the disjunction is true in every context in $\mathcal{K}$. On the other hand, the disjunction of any two of the three clauses does satisfy EX4. As we add more and more potential causes of the fire (explosions, spontaneous combustion, ...) larger and larger disjunctions will count as explanations. The only thing that is not allowed is the disjunction of all possible causes. This distinction between the dijsunction of all potential causes and the disjunction of all but one of the potential causes seems artificial, to say the least. To make matters worse, there is the technical problem of reformulating the minimality condition EX3 to deal with disjunctions. We could not see any reasonable way to deal with these technical problems, so we ban disjunctive explanations.

We believe that, in cases where disjunctive explanations seem appropriate, it is best to capture this directly in the causal model by having a variable that represents the disjunction. (Essentially the same point is made by Chajewska and Halpern [1997].) For example, consider the disjunctive scenario of the arsonist example, where there are other potential causes of the fire. If we want to allow "there was an arsonist" to be an explanation without specifically mentioning who the arsonist is, then it can be easily accomplished by replacing the variables $M L_{1}$ and $M L_{2}$ in the model by a variable $M L$ which is 1 iff at least one arsonist drops a match. Then $M L=1$ becomes an explanation, without requiring disjunctive explanations.

One other point regarding the definition of explanation: When we ask for an explanation of $\varphi$, we usually expect that it not only explains $\varphi$, but that it is true in the actual world. Definition 3.1 makes no reference to the "actual world", only to the agent's epistemic state. There is no difficulty adding an actual world to the picture and requiring that the explanation be true in the world. We can simply talk about an explanation relative to a pair $(\mathcal{K}, \vec{u})$, where $\mathcal{K}$ is a set of contexts. Intuitively, $\vec{u}$ describes the context in the actual world. The requirement that the explanation be true in the actual world then becomes $(M, \vec{u}) \models \vec{X}=\vec{x}$. Although we have not made this requirement part of the definition, adding it would have no significant effect on our discussion. Once we have 
the actual world as part of the model, we could also require that $\vec{u} \in \mathcal{K}$. This condition would entail that $\mathcal{K}$ represents the agent's knowledge rather than the agent's beliefs: the actual context is one of the ones the agent considers possible.

\section{Partial Explanations and Explanatory Power}

Not all explanations are considered equally good. Some explanations are more likely than others. One way to define the "goodness" of an explanation is by bringing probability into the picture. Suppose that the agent has a probability on the set $\mathcal{K}$ of possible contexts. In this case, we can consider the probability of the set of contexts where the explanation $\vec{X}=\vec{x}$ is true. For example, if the agent has reason to believe that electric storms are quite common in both May and June, then the set of contexts where $E S=(1,1)$ holds would have greater probability than the set where either $E S=(1,0)$ or $E S=(0,1)$ holds. Thus, $E S=(1,1)$ would be considered a better explanation.

Formally, suppose that there is a probability $\operatorname{Pr}$ on the set $\mathcal{K}$ of possible contexts. Then the probability of explanation $\vec{X}=\vec{x}$ is just $\operatorname{Pr}(\vec{X}=\vec{x})$. While the probability of an explanation clearly captures some important aspects of how good the explanation is, it is only part of the story. The other part concerns the degree to which an explanation fulfills its role (relative to $\varphi$ ) in the various contexts considered. This becomes clearer when we consider partial explanations. The following example, taken from [Gärdenfors 1988], is one where partial explanations play a role.

Example 4.1: Suppose I see that Victoria is tanned and I seek an explanation. Suppose that the causal model includes variables for "Victoria took a vacation in the Canary Islands", "sunny in the Canary Islands", and "went to a tanning salon". The set $\mathcal{K}$ includes contexts for all settings of these variables compatible with Victoria being tanned. Note that, in particular, there is a context where Victoria went both to the Canaries (and didn't get tanned there, since it wasn't sunny) and to a tanning salon. Gärdenfors points out that we normally accept "Victoria took a vacation in the Canary Islands" as a satisfactory explanation of Victoria being tanned and, indeed, according to his definition, it is an explanation. Victoria taking a vacation is not an explanation (relative to the context $\mathcal{K}$ ) in our framework, since there is a context $\vec{u}^{*} \in \mathcal{K}$ where Victoria went to the Canary Islands but it was not sunny, and in $\vec{u}^{*}$ the actual cause of her tan is the tanning salon, not the vacation. Thus, EX2 is not satisfied. However, intuitively, it is "almost" satisfied, since it is satisfied by every context in $\mathcal{K}$ in which Victoria goes to the Canaries but $u^{*}$. The only complete explanation according to our definition is "Victoria went to the Canary Islands and it was sunny." "Victoria went to the Canary Islands" is a partial explanation, in a sense to be defined below.

In Example 4.1, the partial explanation can be extended to a complete explanation by adding a conjunct. But not every partial explanation can be extended to a complete 
explanation. Roughly speaking, the complete explanation may involve exogenous factors, which are not permitted in explanations. Assume, for example, that going to a tanning salon was not an endogenous variable in the model; instead, the model simply had an exogenous variable $U_{s}$ that could make Victoria suntanned even in the absence of sun in the Canary Islands. Likewise, assume that the weather in the Canary Islands was also part of the background context. In this case, Victoria's vacation would still be a partial explanation of her suntan, since the context where it fails to be a cause (no sun in the Canary Islands) is fairly unlikely, but we cannot add conjuncts to this event to totally exclude that context from the agent's realm of possibilities. Indeed, in this model there is no (complete) explanation for Victoria's tan; it is inexplicable! Inexplicable events are not so uncommon, as the following example shows.

Example 4.2: Suppose that the sound on a television works but there is no picture. Furthermore, the only cause of there being no picture that the agent is aware of is the picture tube being faulty. However, the agent is also aware that there are times when there is no picture even though the picture tube works perfectly well-intuitively, there is no picture "for inexplicable reasons". This is captured by the causal network described in Figure 2, where $T$ describes whether or not the picture tube is working ( 1 if it is and 0 if it is not) and $P$ describes whether or not there is a picture ( 1 if there is and 0 if there is not). The exogenous variable $U_{0}$ determines the status of the picture tube: $T=U_{0}$. The

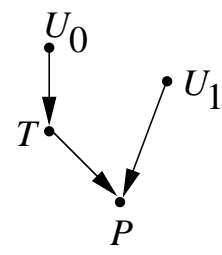

Figure 2: The television with no picture.

exogenous variable $U_{1}$ is meant to represent the mysterious "other possible causes". If $U_{1}=0$, then whether or not there is a picture depends solely on the status of the picture tube - that is, $P=T$. On the other hand, if $U_{1}=1$, then there is no picture $(P=0)$ no matter what the status of the picture tube. Thus, in contexts where $U_{1}=1, T=0$ is not a cause of $P=0$. Now suppose that $\mathcal{K}$ includes a context $\vec{u}_{00}$ where $U_{0}=U_{1}=0$ and $\vec{u}_{10}$ where $U_{0}=1$ and $U_{1}=0$. The only cause of $P=0$ in both $\vec{u}_{00}$ and $\vec{u}_{10}$ is $P=0$ itself. ( $T=0$ is not a cause of $P=0$ in $\vec{u}_{10}$, since $P=0$ even if $T$ is set to 1.) As a result, there is no explanation of $P=0$ relative to an epistemic $\mathcal{K}$ that includes $\vec{u}_{00}$ and $\vec{u}_{10}$. (EX4 excludes the vacuous explanation $P=0$.) On the other hand, $T=0$ is a cause of $P=0$ in all other contexts in $\mathcal{K}$ satisfying $T=0$ other than $\vec{u}_{00}$. If the probability of $\vec{u}_{00}$ (capturing the intuition that it is unlikely that more than one thing goes wrong with a television at once), then we are entitled to view $T=0$ as a quite good partial explanation of $P=0$ with respect to $\mathcal{K}$. 
Note that if we modify the causal model here by adding an endogenous variable, say $I$, corresponding to the "inexplicable" cause $U_{1}$ (with equation $I=U_{1}$ ), then $I=0$ is a cause of $P=0$ in the context $u_{10}$ (and both $I=0$ and $T=0$ are causes of $P=0$ in $\left.u_{00}\right)$. In this model, $I=0$ is an explanation of $P=0$.

Example 4.2 and the discussion after Example 4.1 illustrate an important point. Adding an endogenous variable corresponding to an exogenous variable can result in there being an explanation when there was none before. This phenomenon of adding "names" to create explanations is quite common. For example, "gods" are introduced to explain otherwise inexplicable phenomena; clusters of symptoms are given names in medicine so as to serve as explanations.

In any case, these examples motivate the following definition of partial explanation.

Definition 4.3: Let $\mathcal{K}_{\vec{X}=\vec{x}, \varphi}$ be the largest subset $\mathcal{K}^{\prime}$ of $\mathcal{K}$ such that $\vec{X}=\vec{x}$ is an explanation of $\varphi$ relative to $\mathcal{K}_{\vec{X}=\vec{x}, \varphi}$. (It is easy to see that there is a largest such set; it consists of all the contexts in $\mathcal{K}$ except the ones where $\vec{X}=\vec{x}$ is true but is not a sufficient cause of $\varphi$.) Then $\vec{X}=\vec{x}$ is a partial explanation of $\varphi$ with goodness $\operatorname{Pr}\left(\mathcal{K}_{\vec{X}=\vec{x}, \varphi} \mid \vec{X}=\vec{x}\right)$. Thus, the goodness of a partial explanation measures the extent to which it provides an explanation of $\varphi \cdot{ }^{4}$

In Example 4.1, if the agent believes that it is sunny in the Canary Islands with probability .9 (that is, the probability that it was sunny given that Victoria is suntanned and that she went to the Canaries is .9), then Victoria going to the Canaries is a partial explanation of her being tanned with goodness .9. The relevant set $\mathcal{K}^{\prime}$ consists of those contexts where it is sunny in the Canaries. Similarly, in Example 4.2, if the agent believes that the probability of both the picture tube being faulty and the other mysterious causes being operative is .1 , then $T=0$ is a partial explanation of $P=0$ with goodness .9 (with $\mathcal{K}^{\prime}$ consisting of all the contexts where $U_{1}=1$ ).

A full explanation is clearly a partial explanation with goodness 1 , but we are often satisfied with partial explanations $\vec{X}=\vec{x}$ that are not as good, especially if they have high probability (i.e., if $\operatorname{Pr}(\vec{X}=\vec{x})$ is high). Note that, in general, there is a tension between the goodness of an explanation and its probability.

These ideas also lead to a definition of explanatory power. Consider Example 2.1 yet again, and suppose that there is an endogenous random variable $O$ corresponding to the presence of oxygen. Now if $O=1$ holds in all the contexts that the agent considers possible, then $O=1$ is excluded as an explanation by EX4. If the agent knows that there is oxygen, then the presence of oxygen cannot be part of an explanation. But

\footnotetext{
${ }^{4}$ Here and elsewhere, a formula such as $\vec{X}=\vec{x}$ is being identified with the set of contexts where the formula is true. Recall that, since all contexts in $\mathcal{K}$ are presumed to satisfy $\varphi$, there is no need to condition on $\varphi$; this probability is already updated with the truth of the explanandum $\varphi$. Finally, note that our usage of partial explanation is related to, but different from, that of Chajewska and Halpern [1997].
} 
suppose that $O=0$ holds in one context that the agent considers possible, albeit a very unlikely one (for example, there may be another combustible gas). In that case, $O=1$ becomes a very good partial explanation of the fire. Nevertheless, it is an explanation with, intuitively, very little explanatory power. How can we make this precise?

Suppose that there is a probability distribution $\operatorname{Pr}^{-}$on a set $\mathcal{K}^{-}$of contexts that includes $\mathcal{K}$. $\mathrm{Pr}^{-}$intuitively represents the agent's "pre-observation" probability, that is, the agent's prior probability before the explanandum $\varphi$ is observed or discovered. Thus, $\operatorname{Pr}$ is the result of conditioning $\operatorname{Pr}^{-}$on $\varphi$ and $\mathcal{K}$ consists of the contexts in $\mathcal{K}^{-}$ that satisfy $\varphi$. Gärdenfors identifies the explanatory power of the (partial) explanation $\vec{X}=\vec{x}$ of $\varphi$ with $\operatorname{Pr}^{-}(\varphi \mid \vec{X}=\vec{x}$ ) (see [Chajewska and Halpern 1997; Gärdenfors 1988]). If this probability is higher than $\operatorname{Pr}^{-}(\varphi)$, then the explanation makes $\varphi$ more likely. ${ }^{5}$ Note that since $\mathcal{K}$ consists of all the contexts in $\mathcal{K}^{-}$where $\varphi$ is true, Gärdenfors' notion of explanatory power is equivalent to $\operatorname{Pr}^{-}(\mathcal{K} \mid \vec{X}=\vec{x})$.

Gärdenfors' definition clearly captures some important features of our intuition. For example, under reasonable assumptions about $\operatorname{Pr}^{-}, O=1$ has much lower explanatory power than, say, $M L_{1}=1$. Learning that there is oxygen in the air certainly has almost no effect on an agent's prior probability that the forest burns down, while learning that an arsonist dropped a match almost certainly increases it. However, Gärdenfors' definition still has a problem. It basically confounds correlation with causation. For example, according to this definition, the barometer falling is an explanation of it raining with high explanatory power.

We would argue that a better measure of the explanatory power of $\vec{X}=\vec{x}$ is $\operatorname{Pr}^{-}\left(\mathcal{K}_{\vec{X}=\vec{x}, \varphi} \mid \vec{X}=\right.$ $\vec{x}$ ). Note that the two definitions agree in the case that $\vec{X}=\vec{x}$ is a full explanation (since then $\mathcal{K}_{\vec{X}=\vec{x}, \varphi}$ is just $\mathcal{K}$, the set of contexts in $\mathcal{K}^{-}$where $\varphi$ is true). In particular, they agree that $O=1$ has very low explanatory power, while $M L_{1}=1$ has high explanatory power. The difference between the two definitions arises if there are contexts where $\varphi$ and $\vec{X}=\vec{x}$ both happen to be true, but $\vec{X}=\vec{x}$ is not a cause of $\varphi$. In Example 4.1 , the context $\vec{u}^{*}$ is one such context, since in $\vec{u}^{*}$, Victoria went to the Canary Islands, but this was not an explanation of her getting tanned, since it was not sunny. Because of this difference, for us, the falling barometer has 0 explanatory power as far as explaining the rain. Even though the barometer falls in almost all contexts where it rains (assume that there are contexts where it rains and the barometer does not fall, perhaps because it is defective, so that the barometer falling at least satisfies EX4), the barometer falling is not a cause of the rain in any context. Making the barometer rise would not result in the rain stopping!

Again, (partial) explanations with higher explanatory power typically are more refined

${ }^{5}$ Actually, for Gärdenfors, the explanatory power of $\vec{X}=\vec{x}$ is $\operatorname{Pr}^{-}(\varphi \mid \vec{X}=\vec{x})-\operatorname{Pr}^{-}(\varphi)$. But as far as comparing the explanatory power of two explanations, it suffices to consider just $\operatorname{Pr}^{-}(\varphi \mid \vec{X}=\vec{x})$, since the $\operatorname{Pr}^{-}(\varphi)$ terms will appear in both expressions. We remark that Chajewska and Halpern [1997] argued that the quotient $\operatorname{Pr}^{-}(\varphi \mid \vec{X}) / \operatorname{Pr}^{-}(\varphi)$ gives a better measure of explanatory power than the difference, but the issues raised by Chajewska and Halpern are irrelevant to our concerns here. 
and, hence, less likely, than explanations with less explanatory power. There is no obvious way to resolve this tension. (See [Chajewska and Halpern 1997] for more discussion of this issue.)

As this discussion suggests, our definition shares some features with that of Gärdenfors' [1988]. Like him, we consider explanation relative to an agent's epistemic state. Gärdenfors also considers a "contracted" epistemic state characterized by the distribution $\mathrm{Pr}^{-}$. Intuitively, $\mathrm{Pr}^{-}$describes the agent's beliefs before discovering $\varphi$. (More accurately, it describes an epistemic state as close as possible to Pr where the agent does not ascribe probability 1 to $\varphi$.) If the agent's current epistemic state came about as a result of observing $\varphi$, then we can take $\operatorname{Pr}$ to be the result of conditioning $\operatorname{Pr}^{-}$on $\varphi$. However, Gärdenfors does necessarily assume such a connection between $\operatorname{Pr}$ and $\operatorname{Pr}^{-}$. In any case, for Gärdenfors, $\vec{X}=\vec{x}$ is an explanation of $\varphi$ relative to $\operatorname{Pr}$ if $(1) \operatorname{Pr}(\varphi)=1,(2)$ $0<\operatorname{Pr}(\vec{X}=\vec{x})<1$, and $(3) \operatorname{Pr}^{-}(\varphi \mid \vec{X}=\vec{x})>\operatorname{Pr}^{-}(\varphi)$. (1) is the probabilistic analogue of EX1. Clearly, (2) is the probabilistic analogue of EX4. Finally, (3) says that learning the explanation increases the likelihood of $\varphi$. Gärdenfors focuses on the explanatory power of an explanation, but does not take into account its prior probability. As pointed out by Chajewska and Halpern [1997], Gärdenfors' definition suffers from another defect: If there is an explanation of $\vec{Y}=\vec{y}$ at all, then for all events $\vec{X}=\vec{x}$ such that $0<\operatorname{Pr}(\vec{X}=\vec{x} \wedge \vec{Y}=\vec{y})<1, \vec{X}=\vec{x} \wedge \vec{Y}=\vec{y}$ is an explanation of $\vec{Y}=\vec{y}$. Moreover, it has the highest possible explanatory power. (Note that, in our definition, EX3 blocks $\vec{X}=\vec{x} \wedge \vec{Y}=\vec{y}$ from being a cause of $\vec{Y}=\vec{y}$.)

In contrast to Gärdenfors' definition, the dominant approach to explanation in the AI literature, the maximum a posteriori (MAP) approach (see, for example, [Henrion and Druzdzel 1990; Pearl 1988; Shimony 1991]), focuses on the probability of the explanation, that is, what we have denoted $\operatorname{Pr}(\vec{X}=\vec{x}){ }^{6}$ The MAP approach is based on the intuition that the best explanation for an observation is the state of the world (in our setting, the context) that is most probable given the evidence. There are various problems with this approach (see [Chajewska and Halpern 1997] for a critique). Most of them can be dealt with, except for the main one: it simply ignores the issue of explanatory power. An explanation like $O=1$ has a very high probability, even though it is intuitively irrelevant to the forest burning down. To remedy this problem, more intricate combinations of the quantities $\operatorname{Pr}(\vec{X}=\vec{x}), \operatorname{Pr}^{-}(\varphi \mid \vec{X}=\vec{x})$, and $\operatorname{Pr}^{-}(\varphi)$ have been suggested to quantify the causal relevance of $\vec{X}=\vec{x}$ on $\varphi$ but, as argued by Pearl [2000, p. 221], without taking causality into account, no such combination of parameters can work.

\section{The General Definition}

In general, an agent may be uncertain about the causal model, so an explanation will have to include information about it. (Gärdenfors [1988] and Hempel [1965] make sim-

\footnotetext{
${ }^{6}$ The MAP literature typically considers $\operatorname{Pr}^{-}(\vec{X}=\vec{x} \mid \varphi)$, but this is equivalent to $\operatorname{Pr}(\vec{X}=\vec{x})$.
} 
ilar observations, although they focus not on causal information, but on statistical and nomological information; we return to this point below.) It is relatively straightforward to extend our definition of explanation to accommodate this provision. Now an epistemic state $\mathcal{K}$ consists not only of contexts, but of pairs $(M, \vec{u})$ consisting of a causal model $M$ and a context $\vec{u}$. (Recall that such a pair is a situation.) Intuitively, now an explanation should consist of some causal information (such as "prayers do not cause fires") and the facts that are true. Thus, a (general) explanation has the form $(\psi, \vec{X}=\vec{x})$, where $\psi$ is an arbitrary formula in our causal language and, as before, $\vec{X}=\vec{x}$ is a conjunction of primitive events. We think of the $\psi$ component as consisting of some causal information (such as "prayers do not cause fires", which corresponds to a conjunction of statements of the form $(F=i) \Rightarrow[P \leftarrow x](F=i)$, where $P$ is a random variable describing whether or not prayer takes place). The first component in a general explanation is viewed as restricting the set of causal models. To make this precise, given a causal model $M$, we say $\psi$ is valid in $M$, and write $M \models \psi$, if $(M, \vec{u}) \models \psi$ for all contexts $\vec{u}$ consistent with $M$. With this background, it is easy to state the general definition.

Definition 5.1: $(\psi, \vec{X}=\vec{x})$ is an explanation of $\varphi$ relative to a set $\mathcal{K}$ of situations if the following conditions hold:

EX1. $(M, \vec{u}) \models \varphi$ for each situation $(M, \vec{u}) \in \mathcal{K}$.

EX2. $\vec{X}=\vec{x}$ is a sufficient cause of $\varphi$ in $(M, \vec{u})$ for all $(M, \vec{u}) \in \mathcal{K}$ such that $(M, \vec{u}) \models$ $\vec{X}=\vec{x}$ and $M \models \psi \cdot \vec{X}=\vec{x}$ is a sufficient cause of $\varphi$ in $(M, \vec{u})$.

EX3. $(\psi, \vec{X}=\vec{x})$ is minimal; there is no pair $\left(\psi^{\prime}, \vec{X}^{\prime}=\vec{x}^{\prime}\right) \neq(\psi, \vec{X}=\vec{x})$ satisfying EX2 such that $\left\{M^{\prime \prime} \in M(\mathcal{K}): M^{\prime \prime} \models \psi^{\prime}\right\} \supseteq\left\{M^{\prime \prime} \in M(\mathcal{K}): M^{\prime \prime} \models \psi\right\}$, where $\mathcal{M}(\mathcal{K})=\{M:(M, \vec{u}) \in \mathcal{K}$ for some $\vec{u}\}, \vec{X}^{\prime} \subseteq \vec{X}$, and $\vec{x}^{\prime}$ is the restriction of $\vec{x}$ to the variables in $\vec{X}^{\prime}$. Roughly speaking, this says that no subset of $X$ provides a sufficient cause of $\varphi$ in more contexts than those where $\psi$ is valid.

$\operatorname{EX4.~}(M, \vec{u}) \models \neg(\vec{X}=\vec{x})$ for some $(M, \vec{u}) \in \mathcal{K}$ and $\left(M^{\prime}, \vec{u}^{\prime}\right) \models \vec{X}=\vec{x}$ for some $\left(M^{\prime}, \vec{u}^{\prime}\right) \in$ $\mathcal{K}$.

Note that, in EX2, we now restrict the requirment of sufficiency to situations $(M, \vec{u}) \in \mathcal{K}$ that satisfy both parts of the explanation $(\psi, \vec{X}=\vec{x})$, in that $M \models \psi$ and $(M, \vec{u}) \models \vec{X}=$ $\vec{x}$. Furthermore, although both components of an explanation are formulas in our causal language, they play very different roles. The first component serves to restrict the set of causal models considered (to those with the appropriate structure); the second describes a cause of $\varphi$ in the resulting set of situations.

Clearly Definition 3.1 is the special case of Definition 5.1 where there is no uncertainty about the causal structure (i.e., there is some $M$ such that if $\left(M^{\prime}, \vec{u}\right) \in \mathcal{K}$, then $\left.M=M^{\prime}\right)$. In this case, it is clear that we can take $\psi$ in the explanation to be true. 
Example 5.2: Using this general definition of causality, let us consider Scriven's [1959] famous paresis example, which has caused problems for many other formalisms. Paresis develops only in patients who have been syphilitic for a long time, but only a small number of patients who are syphilitic in fact develop paresis. Furthermore, according to Scriven, no other factor is known to be relevant in the development of paresis. ${ }^{7}$ This description is captured by a simple causal model $M_{P}$. There are two endogenous variables, $S$ (for syphilis) and $P$ (for paresis), and two exogenous variables, $U_{1}$, the background factors that determine $S$, and $U_{2}$, which intuitively represents "disposition to paresis", that is, the factors that determine, in conjunction with syphilis, whether paresis actually develops. An agent who knows this causal model and that a patient has paresis does not need an explanation of why: he knows without being told that the patient must have syphilis and that $U_{2}=1$. On the other hand, for an agent who does not know the causal model (i.e., considers a number of causal models of paresis possible), $\left(\psi_{P}, S=1\right)$ is an explanation of paresis, where $\psi_{P}$ is a formula that characterizes $M_{P}$.

Definition 5.1 can also be extended to deal naturally with probability. Actually, probability plays a role in two places. First, there is a probability on the situations in $\mathcal{K}$, analogous to the probability on contexts discussed in Section 4. Using this probability, it is possible to talk about the goodness of a partial explanation and to talk about explanatory power, just as before. In addition, probability can also be added to causal models to get probabilistic causal models. A probabilistic causal model is a tuple $M=$ $(\mathcal{S}, \mathcal{F}, \operatorname{Pr})$, where $M=(\mathcal{S}, \mathcal{F})$ is a causal model and $\operatorname{Pr}$ is a probability measure on the contexts defined by signature $\mathcal{S}$ of $M$. In a probabilistic causal model, it is possible to talk about the probability that $X=3$; this is just the probability of the set of context in $M$ where $X=3$. More importantly for our purposes, it is also possible to say "with probability .9, $X=3$ causes $Y=1$ ". Again, this is just the probability of the set of contexts where $X=3$ is the cause of $Y=1$.

Once we allow probabilistic causal models, explanations can include statements like "with probability .9, working with asbestos causes lung cancer". Thus, probabilistic causal models can capture statistical information of the kind considered by Gärdenfors and Hempel. To fit such statements into the framework discussed above, we must extend the language to allow statements about probability. For example, $\operatorname{Pr}([X \leftarrow 3](Y=1))=$ .9 , "the probability that setting $X$ to 3 results in $Y$ being 1 ", would be a formula in the language. Such formulas could then become part of the first component $\psi$ in a general explanation. ${ }^{8}$

\footnotetext{
${ }^{7}$ Apparently there are now other known factors, but this does not change the import of the example.

${ }^{8}$ We remark that Gärdenfors also consider two types of probability measures, one on his analogue of situations, and one on the worlds in a model. Like here, the probability measure on worlds in a model allows explanations with statistical information, while the probability on situations allows him to define his notion of explanatory power.
} 


\section{Discussion}

We have given a formal definition of explanation in terms of causality. As we mentioned earlier, there are not too many formal definitions of explanation in terms of causality in the literature. One of the few exceptions is given by Lewis [1986], who defends the thesis that "to explain an event is to provide some information about its causal history". While this view is compatible with our definition, there is no formal definition given to allow for a careful comparison between the approaches. In any case, if we were to define causal history in terms of Lewis's [1973] definition of causality, we would inherit all the problems of that definition. Our definition avoids these problems.

We have mentioned one significant problem of the definition already: dealing with disjunctive explanations. Disjunctions cause problems in the definition of causality, which is why we do not deal with them in the context of explanation. As we pointed out earlier, it may be possible to modify the definition of causality so as to be able to deal with disjunctions without changing the structure of our definition of explanation. In addition, our definition gives no tools for dealing with the inherent tension between explanatory power, goodness of partial beliefs, and the probability of the explanation. Clearly this is an area that requires further work.

\section{A Appendix: The Formal Definition of Causality}

To keep this part of the paper self-contained, we reproduce here the formal definition of actual causality from Part I.

Definition A.1: (Actual cause) $\vec{X}=\vec{x}$ is an actual cause of $\varphi$ in $(M, \vec{u})$ if the following three conditions hold:

AC1. $(M, \vec{u}) \models(\vec{X}=\vec{x}) \wedge \varphi$. (That is, both $\vec{X}=\vec{x}$ and $\varphi$ are true in the actual world.)

AC2. There exists a partition $(\vec{Z}, \vec{W})$ of $\mathcal{V}$ with $\vec{X} \subseteq \vec{Z}$ and some setting $\left(\vec{x}^{\prime}, \vec{w}^{\prime}\right)$ of the variables in $(\vec{X}, \vec{W})$ such that if $(M, \vec{u}) \models\left(Z=z^{*}\right)$ for all $Z \in \vec{Z}$, then

(a) $(M, \vec{u}) \models\left[\vec{X} \leftarrow \vec{x}^{\prime}, \vec{W} \leftarrow \vec{w}^{\prime}\right] \neg \varphi$. In words, changing $(\vec{X}, \vec{W})$ from $(\vec{x}, \vec{w})$ to $\left(\vec{x}^{\prime}, \vec{w}^{\prime}\right)$ changes $\varphi$ from true to false;

(b) $(M, \vec{u}) \models\left[\vec{X} \leftarrow \vec{x}, \vec{W}^{\prime} \leftarrow \vec{w}^{\prime}, \vec{Z}^{\prime} \leftarrow \vec{z}^{*}\right] \varphi$ for all subsets $\vec{W}^{\prime}$ of $\vec{W}$ and all subsets $\vec{Z}^{\prime}$ of $\vec{Z}$. In words, setting any subset of variables in $\vec{W}$ to their values in $\vec{w}^{\prime}$ should have no effect on $\varphi$ as long as $\vec{X}$ is kept at its current value $\vec{x}$, even if all the variables in an arbitrary subset of $\vec{Z}$ are set to their original values in the context $\vec{u}$.

AC3. $\vec{X}$ is minimal; no subset of $\vec{X}$ satisfies conditions AC1 and AC2. Minimality ensures that only those elements of the conjunction $\vec{X}=\vec{x}$ that are essential for changing $\varphi$ in $\mathrm{AC} 2(\mathrm{a})$ are considered part of a cause; inessential elements are pruned. 
$\vec{X}=\vec{x}$ is a sufficient cause of $\varphi$ in $(M, \vec{u})$ if $\mathrm{AC} 1$ and $\mathrm{AC} 2$ hold, but not necessarily AC3.

We remark that in Part I, a slight generalization of this definition is considered. Rather than allowing all settings of $\vec{X}$ and $\vec{W}$ in AC2, the more general definition presupposes a set of allowable settings. All the settings used in AC2 must come from this allowable set. It is shown that by generalizing in this way, it is possible to avoid a number of problematic examples.

\section{Acknowledgments:}

Thanks to Riccardo Pucella and Vicky Weissman for useful comments.

\section{References}

Chajewska, U. and J. Y. Halpern [1997]: Defining explanation in probabilistic systems. In Proc. Thirteenth Conference on Uncertainty in Artificial Intelligence (UAI '97), pp. $62-71$.

Eiter, T. and T. Lukasiewicz [2002]: Complexity results for structure-based causality. Artificial Intelligence 142:1, pp. 53-89.

Gärdenfors, P. [1988]: Knowledge in Flux. Cambridge, Mass.: MIT Press.

Goldberger, A. S. [1972]: Structural equation methods in the social sciences. Econometrica 40:6, pp. 979-1001.

Halpern, J. Y. and J. Pearl [2004]: Causes and explanations: A structural-model approach. Part I: Causes. British Journal for Philosophy of Science.

Hempel, C. G. [1965]: Aspects of Scientific Explanation. Free Press.

Henrion, M. and M. J. Druzdzel [1990]: Qualitative propagation and scenario-based approaches to explanation of probabilistic reasoning. In Uncertainty in Artificial Intelligence 6, pp. 17-32.

Hopkins, M. [2001]: A proof of the conjunctive cause conjecture. Unpublished manuscript.

Lewis, D. [1986]: Causal explanation. In Philosophical Papers, Volume II, pp. 214-240. New York: Oxford University Press.

Lewis, D. K. [1973]: Counterfactuals. Cambridge, Mass.: Harvard University Press.

Pearl, J. [1988]: Probabilistic Reasoning in Intelligent Systems. San Francisco: Morgan Kaufmann.

Pearl, J. [1995]: Causal diagrams for empirical research. Biometrika 82:4, pp. 669-710. 
Pearl, J. [2000]: Causality: Models, Reasoning, and Inference. New York: Cambridge University Press.

Salmon, W. C. [1989]: Four Decades of Scientific Explanation. Minneapolis: University of Minnesota Press.

Scriven, M. J. [1959]: Explanation and prediction in evolutionary theory. Science 130, pp. $477-482$.

Shimony, S. E. [1991]: Explanation, irrelevance and statistical independence. In Proceedings, Ninth National Conference on Artificial Intelligence (AAAI '91), pp. 482487.

Sosa, E. and M. Tooley (Eds.) [1993]: Causation. Oxford readings in Philosophy. Oxford: Oxford University Press.

Woodward, J. [2001]: Explanation. In The Blackwell Guide to the Philosophy of Science. To appear. 